\title{
Video Article \\ Imine Metathesis by Silica-Supported Catalysts Using the Methodology of Surface Organometallic Chemistry
}

\author{
Maha A. Aljuhani ${ }^{1}$, Jérémie D.A. Pelletier ${ }^{1}$, Jean-Marie Basset ${ }^{1}$ \\ ${ }^{1}$ KAUST Catalysis Center, Division of Physical Sciences and Engineering, King Abdullah University of Science and Technology (KAUST)
}

Correspondence to: Jérémie D.A. Pelletier at jeremie.pelletier@kaust.edu.sa

URL: https://www.jove.com/video/59409

DOI: doi:10.3791/59409

Keywords: Chemistry, Issue 152, surface organometallic chemistry (SOMC), metal-nitrogen fragments, catalysis by design, heterogeneous catalysis, dehydroxylated silica, imine metathesis, well-defined single-site catalysts

Date Published: 10/18/2019

Citation: Aljuhani, M.A., Pelletier, J.D., Basset, J.M. Imine Metathesis by Silica-Supported Catalysts Using the Methodology of Surface Organometallic Chemistry. J. Vis. Exp. (152), e59409, doi:10.3791/59409 (2019).

\section{Abstract}

With this protocol, a well-defined singlesite silica-supported heterogeneous catalyst $\left[(\equiv \mathrm{Si}-\mathrm{O}-) \mathrm{Hf}(=\mathrm{NMe})\left(\eta^{1}-\mathrm{NMe}_{2}\right)\right]$ is designed and prepared according to the methodology developed by surface organometallic chemistry (SOMC). In this framework, catalytic cycles can be determined by isolating crucial intermediates. All air-sensitive materials are handled under inert atmosphere (using gloveboxes or a Schlenk line) or high vacuum lines (HVLs, $<10^{-5} \mathrm{mbar}$ ). The preparation of $\mathrm{SiO}_{2-700}$ (silica dehydroxylated at $700{ }^{\circ} \mathrm{C}$ ) and subsequent applications (the grafting of complexes and catalytic runs) requires the use of HVLs and double-Schlenk techniques. Several well-known characterization methods are used, such as Fourier-transform infrared spectroscopy (FTIR), elemental microanalysis, solid-state nuclear magnetic resonance spectroscopy (SSNMR), and state-of-the-art dynamic nuclear polarization surface enhanced NMR spectroscopy (DNP-SENS). FTIR and elemental microanalysis permit scientists to establish the grafting and its stoichiometry. ${ }^{1} \mathrm{H}$ and ${ }^{13} \mathrm{C}$ SSNMR allows the structural determination of the hydrocarbon ligands coordination sphere. DNP SENS is an emerging powerful technique in solid characterization for the detection of poorly sensitive nuclei ( ${ }^{15} \mathrm{~N}$, in our case). $\mathrm{SiO}_{2-700}$ is treated with about one equivalent of the metal precursor compared to the amount of surface silanol $\left(0.30 \mathrm{mmol} \cdot \mathrm{g}^{-1}\right)$ in pentane at room temperature. Then, volatiles are removed, and the powder samples are dried under dynamic high vacuum to afford the desired materials $\left[(\equiv \mathrm{Si}-\mathrm{O}-) \mathrm{Hf}\left(\eta^{2} \pi-\mathrm{MeNCH}_{2}\right)\left(\eta^{1}-\mathrm{NMe}_{2}\right)\left(\eta^{1}-\mathrm{HNMe}_{2}\right)\right]$. After a thermal treatment under high vacuum, the grafted complex

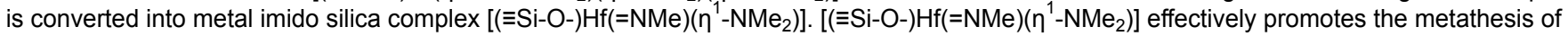
imines, using the combination of two imine substrates, $\mathrm{N}$-(4-phenylbenzylidene)benzylamine, or N-(4-fluorobenzylidene)-4-fluoroaniline, with $\mathrm{N}$ benzylidenetert-butylamine as substrates. A significantly lower conversion is observed with the blank runs; thus, the presence of the imido group in $\left[(\equiv \mathrm{Si}-\mathrm{O}-) \mathrm{Hf}(=\mathrm{NMe})\left(\eta^{1}-\mathrm{NMe}_{2}\right)\right]$ is correlated to the catalytic performance.

\section{Video Link}

The video component of this article can be found at https://www.jove.com/video/59409/

\section{Introduction}

SOMC has a rich library of surface complexes active for a wide range of catalytic reactions and an unparalleled track record to isolate the catalytic intermediates in heterogeneous catalysis. Single-site catalysts have been prepared by the reaction of an organometallic compound (or a coordination compound) with a very clean surface of highly dehydroxylated metal oxide (e.g., silica). Recently, we have identified surface organometallic fragments (SOMF) (e.g., $\mathrm{M}=\mathrm{C}, \mathrm{M}-\mathrm{C}, \mathrm{M}-\mathrm{H}, \mathrm{M}-\mathrm{NC}, \mathrm{M}=\mathrm{O}, \mathrm{M}=\mathrm{CR}_{2}$, andM=NR) that have a pivotal role toward targeted catalysis reactivity (e.g., alkane oxidation ${ }^{1,2,3}$, alkane metathesis ${ }^{4,5}$, alkene metathesis ${ }^{6}$, imine metathesis ${ }^{7,8,9}$ ). $[M]=N R$ fragments have received less attention; their characterization and reactivity studies remain limited, yet they can act as an intermediate in carbon-nitrogen transformation reactions ${ }^{10}$. The importance of imido complexes (bearing a $[M]=N R$ ) is their well-established organic synthesis and catalysis ${ }^{11,12}$. Stoichiometric or catalytic reactions may occur at the $M=N R$ fragment itself ${ }^{13}$, or the imido group may remain a spectator, as in Schrock's catalysts for olefin metathesis ${ }^{14}$ or some Ziegler-Natta-type olefin polymerization catalysts ${ }^{15}$. This paper deals with a reaction of imine metathesis with a group IV organometallic complex bearing a metallaaziridine fragment which transfers to a metal-imido fragment that promotes the catalysis ${ }^{16}$. Catalytic imine metathesis is analogous to olefin metathesis in that two different imines afford a statistical mixture of all possible $=$ NR exchange products

Metathesis reactions were discovered in $1964^{17}$ by Phillips Petroleum Co., Bartlesville, Oklahoma. A variety of heterogeneous catalysts were developed for olefin metathesis (e.g., tungsten, molybdenum oxide on silica or alumina, or rhenium oxide on alumina) ${ }^{18}$. Most progresses were reported in olefin metathesis ${ }^{19,20}$ and advanced the general understanding of hydrocarbon transformations. They were recognized in 2005 by the award of the Nobel Prize to scientists who worked in olefin metathesis, namely Richard R. Schrock, Yves Chauvin, and Robert Grubbs ${ }^{21}$. The chemistry of metal-alkylidene-mediated olefin metathesis processes has broadened not only pure hydrocarbon organic synthesis possibilities ${ }^{22}$ but also made it possible to deliver chemicals with new carbon-heteroatom double bonds ${ }^{7,16}$. Alkane metathesis was discovered later by Basset's group and requires multifunctional catalysts ${ }^{23,24,25}$. Less attention has been dedicated to imine metathesis, yet it can be a promising route to a variety of nitrogen-containing compounds. 
Metal-mediated catalytic systems can selectively metathesize carbon-nitrogen bonds ${ }^{26,27,28}$. Homogeneous catalytic systems have been employed for imine metathesis, yet no definite mechanism has been validated ${ }^{29}$. This has hindered the rational development of new efficient synthetic pathways to yield new compounds. A practical perspective developing a metal-catalyzed imine metathesis may improve its selectivity and its tolerances toward functional groups compared to acid-catalyzed imine exchange ${ }^{30,31}$.

Employing rigorous SOMC methodology, we have isolated two new well-defined hafnium nitrogen-containing fragments (metallaaziridine and metal-imido) that were fully characterized (FTIR, solid-state NMR, elemental microanalysis) ${ }^{7}$. Using highly dehydroxylated silica with isolated silanols, $\mathrm{SiO}_{2-700}$ (Figure 1) allows the isolation of well-defined single-site catalysts. This work is considering the $\mathrm{M}-\mathrm{N}, \mathrm{MNC}$, and $\mathrm{M}=\mathrm{N}$ surface fragments (SFs) as key reaction intermediates that direct the reaction toward hydroaminoalkylation ${ }^{26}$ or imine metathesis ${ }^{7,8,32}$. This study can potentially bring a better general understanding of transition metal imido functional groups bore by group IV metal grafted on silica.

One major issue with a heteroatom containing SFs, including nitrogen ones, has been the limited amount of characterization methods available to identify the metal-heteroatom coordination. This work is showing that ${ }^{15} \mathrm{~N}$ DNP-SENS can provide a clear insight into the silica-supported nitrogen moieties (metallaaziridine and metal-imido). Herein, we isolate a silica-supported well-defined (imido) complex and demonstrate its capability as a highly efficient imine metathesis catalyst ${ }^{7,33}$.

Overall, this work leads to an improved understanding of the imine metathesis mechanism catalyzed by surface complexes. This protocol can be generalized to other early transition metal imine complexes grafted on highly dehydroxylated silica surfaces. Those surface species could be employed as catalysts but only for the hydroamination and hydroaminoalkylation of alkenes or alkynes ${ }^{26,34,35,36,37}$, but also for the metathesis of imines representing a $2+2$ mechanism including the imine and the imido groups see (Figure 2). Imine products are valuable in pharmaceutical and agricultural applications ${ }^{35,38}$.

\section{Protocol}

\section{Leak check for all the high vacuum vessels and their connection to the HVL}

1. Connect the vessel to the HVL.

2. Check whether the pressure increases by alternating the dynamic and the static vacuum.

3. In case of a leak, scan the connection with the high-frequency generator to localize leaks and holes.

\section{Dehydroxylated silica preparation}

1. Cover fumed silica $(5 \mathrm{~g}$ in a $100 \mathrm{~mL}$ beaker) with enough deionized water (ca. $50 \mathrm{~mL}$ ) till it becomes a compact gel.

2. Cover the beaker with some aluminum foil and heat it in the oven at $200{ }^{\circ} \mathrm{C}$ overnight.

3. Grind the silica and transfer $(1 \mathrm{~g})$ to a glass reactor $(30 \mathrm{~cm}$ in height, with a diameter of $4 \mathrm{~cm})$; then, close it with a cap and seal it with grease.

4. Connect the glass reactor to the port of the HVL.

5. Start heating it gradually to reach $700{ }^{\circ} \mathrm{C}$ and leave it overnight.

6. Let the reactor cool down and transfer it to the glovebox.

7. Prepare a disc pellet from the silica dehydroxylated at $700{ }^{\circ} \mathrm{C}$ for a transmission FTIR measurement in the glovebox.

8. Observe the isolated silanol signal in the FTIR spectrum.

\section{Solvent preparation (removal of oxygen and moisture)}

1. Prepare sodium-mirror-coated solvent bomb equipped with a Teflon stopcok.

2. Transfer pentane to the solvent bomb.

3. Connect the solvent bomb to the HVL.

4. Check for leaks in the connections.

5. Freeze the solvent using a liquid-nitrogen-filled dewar.

6. Evacuate till the solvent finishes to degas.

7. Stop the evacuation.

8. Repeat the freeze (step 3.5); then, perform a degas cycle (steps 3.6 and 3.7) at least $2 x$ more to ensure all gases are removed.

\section{General procedure for grafting metal complexes on dehydroxylated silica using $\mathrm{Hf}\left(\mathrm{NMe}_{2}\right)_{4}$}

1. Dry a double Schlenk by evacuating it with the HVL and heating it with a heat gun

2. Put the dry double Schlenk into the glovebox.

3. In the double Schlenk, add the precursor complex $\mathrm{Hf}\left(\mathrm{NMe}_{2}\right)_{4}(0.33 \mathrm{mmol}, 0.089 \mathrm{~mL})$ to one compartment and the dehydroxylated silica (1 $\left.\mathrm{g}\right)$ to the other side with a stir bar.

4. Close the two necks of the double Schlenk with cap and seal them with grease.

5. Using a T-joint, connect the HVL to the solvent Schlenk on one side and to the double Schlenk on the other side.

6. Ensure that all connections are secured by metallic clips and evacuate the line and the double Schlenk until reaching a stable high vacuum $\left(10^{-5} \mathrm{mbar}\right)$. All stopcocks should be evacuated.

7. Transfer the solvent from the solvent Schlenk to the compartment of the double Schlenk containing the metal precursor by distillation. Once the glassware assembly is under static vacuum, use a liquid nitrogen dewar to cool the compartment to condense the solvent and to dissolve the precursor there. 
8. Transfer the solution to the silica compartment by gravitation. Stir it for $1-3 \mathrm{~h}$ to complete grafting.

9. Introduce extra solvent by distillation to wash the silica.

10. Start by washing the material; then, filter the material by transferring the solvent to the solvent compartment and distill the solvent to the solid compartment.

11. Remove the waste solvent by distillation using an interceptor (solvent trap). Dry the material by using an HVL with continuous stirring in the beginning; then, leave it under a vacuum overnight.

12. Prepare a disc pellet (50-70 mg of prepared material) for an FTIR measurement in the glovebox.

\section{Preparation of the catalyst}

1. Add the grafted material $(1 \mathrm{~g})$ to a Schlenk. Connect it to the HVL.

2. Start heating it gradually to reach $200{ }^{\circ} \mathrm{C}$ and leave it for $4 \mathrm{~h}$. Leave it to cool down under vacuum.

3. Prepare a disc pellet (50-70 mg of prepared material) for an FTIR measurement in the glovebox.

\section{Imine metathesis catalysis}

1. In an ampule tube (or sealed vial), add the catalyst $(0.0031 \mathrm{mmol}, 12.47 \mathrm{mg}$ ).

2. Add two imine substrates, for example, $\mathrm{N}$-(4-phenylbenzylidene)benzylamine $(0.0783 \mathrm{mmol}, 21.36 \mathrm{mg})$ with $\mathrm{N}$-benzylidene-tert-butylamine (0.0783 mmol, $13.94 \mathrm{uL})$. Or, use $\mathrm{N}$-(4-fluorobenzylidene)-4-fluoroaniline $(0.0783 \mathrm{mmol}, 17.00 \mathrm{mg})$ with $\mathrm{N}$-(4-phenylbenzylidene)benzylamine (0.0783 mmol, $21.36 \mathrm{mg})$.

3. Add $0.5 \mathrm{~mL}$ of toluene. Add a stirring bar. Connect the ampule tube to the vacuum line. Freeze the ampule tube by using liquid nitrogen.

4. Use the flame torch (oxygen and propane) to seal the ampule tube. For a sealed vial, just close it tightly by the compressor.

5. Place the tube in oil or in a sand bath at $80^{\circ} \mathrm{C}$.

6. Leave the experiment run for up to $6 \mathrm{~h}$. Monitor the reaction at different time from 1 to $6 \mathrm{~h}$.

7. Freeze the tube and cut the top using a glass cutter.

8. Filter the solution into a gas chromatography (GC) vial for GC mass spectrometry (GC-MS) analysis. Dilute the reaction solution to a volume of $1 \mathrm{~mL}$.

\section{Representative Results}

Firstly, preparing silica 700 as described in section 2 of the protocol, the fumed silica should be mixed with enough deionized water to make it compact, left overnight in the oven at $120^{\circ} \mathrm{C}$, and then, loaded to a quartz reactor (Figure 3). Dehydroxylated silica $\mathrm{SiO}_{2-700} \mathrm{Was}_{\mathrm{S}} \mathrm{bbtained}$ by heating the silica gradually to $700{ }^{\circ} \mathrm{C}$ under a dynamic vacuum, FTIR spectrum for $\mathrm{SiO}_{2-700}$ in (Figure 1) shows the characteristic isolated silanol of $\mathrm{SiO}_{2-700}$.

The grafting of metal complex to the silica was achieved by a protonolysis reaction of the amido ligands $\left(\mathrm{NMe}_{2}\right)$ by surface silanols. For the grafting of organometallic complexes onto highly dehydroxylated silica surface $\mathrm{SiO}_{2-700}$ (Figure 4), dehydroxylated silica was loaded with complex $\mathrm{Hf}\left(\mathrm{NMe}_{2}\right)_{4}$ into a double Schlenk inside a glovebox. The double Schlenk was moved out of the glovebox for the reaction to occur. The grafting reaction lasted around $3 \mathrm{~h}$. Three washing cycles were then carried out by filtration; more solvent was transferred by distillation when needed. Finally, the solvent was distilled and then removed, with all volatiles, using an interceptor (solvent trap). All solids were dried by connecting the double Schlenk to the HVL (Figure 4 and Figure 5).

Elemental analysis using inductively coupled plasma (ICP) (using the EPA 3052 method for digestion) and carbon, hydrogen, nitrogen, and sulfur analyzer (CHNS) accompanied with FTIR was first verified for the determination of grafting stoichiometry. Elemental analysis showed that N/M $=3.9$ (theory = 3) and $\mathrm{C} / \mathrm{M}=7.1$ (theory = 6) for the grafted material (see Table 1); for 2, the N/M and C/M ratios were 2.5 (theory = 2) and 4.6 (theory $=4$ ), respectively. Thus, the carbon and nitrogen contents were declined. For the preparation of the samples for the FTIR measurement using the designed FTIR cell, see Figure 6. The first sample is the dehydroxylated $\mathrm{SiO}_{2-700}$, which displayed a characteristic peak for isolated silanol. After grafting the complex on dehydroxylated silica, this characteristic peak almost completely disappeared, and new peaks appeared in the alkyl region at $2,776-2,970 \mathrm{~cm}^{-1}$ and $1,422-1,465 \mathrm{~cm}^{1}$. After a heat treatment of prepared material at $200{ }^{\circ} \mathrm{C}$ for $1 \mathrm{~h}$, its infrared (IR) spectrum showed a new peak for the imido fragment at $1,595 \mathrm{~cm}^{-1}$.

SSNMR experiments were carried out for a deeper understanding of the surface structure. Samples for SSNMR (Figure 7) were loaded in a specific rotor $(32.1 \mu \mathrm{L})$. The ${ }^{1} \mathrm{H}$ SSNMR spectrum of the grafted material displayed broad peaks at 2.2 and $2.7 \mathrm{ppm}$ for alkyl groups bonded to the nitrogen ligands. The breadth of the resonances was expected in ${ }^{1} \mathrm{H}$ SSNMR (in contrast to liquid NMR) and was also associated with less mobile surface species (Figure 7B).

The ${ }^{13} \mathrm{C}$ cross-polarized magic angle spinning (CP-MAS) spectrum displayed signals that were less broadened but had a low sensitivity (alike liquid NMR). The spectrum of the grafted material revealed two overlapping peaks at $37 \mathrm{ppm}-\mathrm{N}-\left(\mathrm{CH}_{3}\right)_{2}$ and at $46 \mathrm{ppm}$, attributed to the nonequivalent methyl group in $-\mathrm{N}-\left(\mathrm{CH}_{3}\right)$ alongside a low-intensity peak at $81 \mathrm{ppm}$. A heteronuclear correlation spectroscopy (HETCOR) experiment was carried out to show the correlation between proton and carbon directly bonded to each other. The $81 \mathrm{ppm}$ signal was correlated with the proton peak at $2.7 \mathrm{ppm}$ in the HETCOR spectrum ${ }^{7}$. As previously reported with zirconiaaziridene, this peak represented the methylene $\left(\mathrm{CH}_{2}\right)$ group in a metallaaziridine cycle ${ }^{39}$. 
A multiple-quantum experiment makes it possible to visualize the correlation between proximal protons. Double-quantum (DQ) NMR indicates the sum of the two single quantum NMR frequencies to provide an autocorrelation peak located where $\omega 1=2 \omega 2$. Similarly, for triple-quantum (TQ) NMR impart from the sum of three protons single quantum frequencies where $\omega 1=3 \omega 2$. $\mathrm{CH}_{2}$ and $\mathrm{CH}_{3}$ afford characteristic autocorrelation peaks in the double- and triple-quantum dimensions, respectively. DQ and TQ proton SSNMR experiments were performed with the grafted material. For the strongest autocorrelation peak observed for the signal at $2.2 \mathrm{ppm}$ in both the DQ and TQ spectra (appearing at $4.4 \mathrm{ppm}$ and $6.6 \mathrm{ppm}$ in $\mathrm{DQ}$ and $\mathrm{TQ}$, respectively), revealing $-\mathrm{CH}_{3}$ protons, see Figure 7B. The second overlapping peak for the proton at $2.7 \mathrm{ppm}$ showed an autocorrelation only in the double quantum (DQ) spectrum; thus, it confirms the presence of a methylene group (-CH $\left.2^{-}\right)$in the grafted species.

${ }^{15} \mathrm{~N}$ SSNMR experiments using the DNP-SENS technique (Figure 7B) were carried out to characterize the nitrogen atoms coordinated to the metal center. The spectrum obtained for the grafted material displayed two peaks, around 7 and $32 \mathrm{ppm}$. On the basis of its relative intensity, the intense signal downfield at $32 \mathrm{ppm}$ was assigned to the nitrogen nuclei of the $\left(\eta^{2}-\mathrm{NMeCH}_{2}\right)$ and $\left(-\mathrm{NMe}_{2}\right)$ functionalities. The weak upfield-shifted peak at $7 \mathrm{ppm}$ was attributed to an $\mathrm{NH}\left(\mathrm{CH}_{3}\right)_{2}$ moiety; upon heat treatment, - $-\mathrm{HNMe}_{2}$ remains on the surface.

For the imido metal fragment in catalyst 2, generated after heat treatment, one broad peak appeared at 2.2 ppm, and the weak peaks at 1.2 and $0.7 \mathrm{ppm}$ in the ${ }^{1} \mathrm{H}$ NMR spectrum attributed to some minor impurities, dimethylamine moieties. For ${ }^{13} \mathrm{C}$ CP-MAS, NMR spectrum displays two peaks at 37 and $48 \mathrm{ppm}$. Interestingly, the peak for $\left(-\mathrm{CH}_{2}^{-}\right)$in metallaaziridine disappeared (Figure 7C). Additionally, we inferred from the multiple quantum experiments that the ${ }^{1} \mathrm{H}$ peak appearing at $2.2 \mathrm{ppm}$ represented the $\left(-\mathrm{CH}_{3}\right)$ protons. For the ${ }^{15} \mathrm{~N}$ SSNMR spectrum of the imido metal fragment in $\mathbf{2}$ (Figure 7C), an additional downfield-shifted peak at $113 \mathrm{ppm}$ along with a peak at 34 ppm became much less intense after the heat treatment of the grafted material. The $113 \mathrm{ppm}$ peak was assigned to the new fragment generated from the hafnium imido moieties.

In a glovebox, imine substrates with a catalyst were loaded either in an ampule tube or a sealed vial with toluene, and Figure 8 shows the reaction of imine metathesis with three imine compounds, namely $\mathrm{N}$-(4-phenylbenzylidene)benzylamine, N-(4-fluorobenzylidene)-4fluoroaniline, and N-benzylidenetert-butylamine. Mass spectra for products analyzed by GC-MS (Figure 8) are a" (1-([1,1'-biphenyl]-4-yl)-N(tert-butyl)methanimine), c' (N,1-diphenylmethanimine), a' (1-([1,1'-biphenyl]-4-yl)-N-(3-fluorophenyl)methanimine) and b' (1-(4-fluorophenyl)-Nphenylmethanimine).

Imine metathesis is a combination of two imine substrates mixed with the catalyst to produce a new two imine substrates after characterizing by GC-MS. To calculate the conversion percentage as shown in (Table 2), use the following formulas.
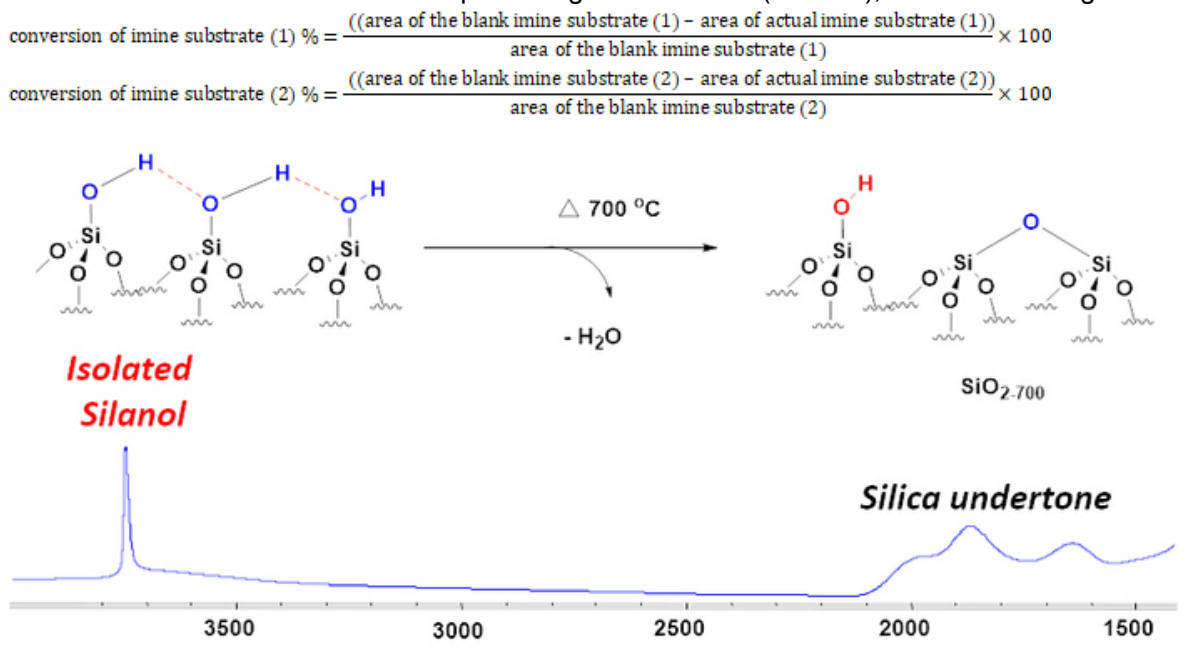

Wavenumbers $\left(\mathrm{cm}^{-1}\right)$

Figure 1: Dehydroxylation. Formation of isolated silanols by dehydroxylation to produce silica dehydroxylated at $700{ }^{\circ} \mathrm{C}\left(\mathrm{SiO}{ }_{2-700}\right) . \mathrm{Please}$ click here to view a larger version of this figure. 
A)

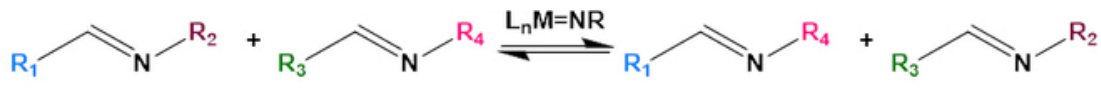

B)

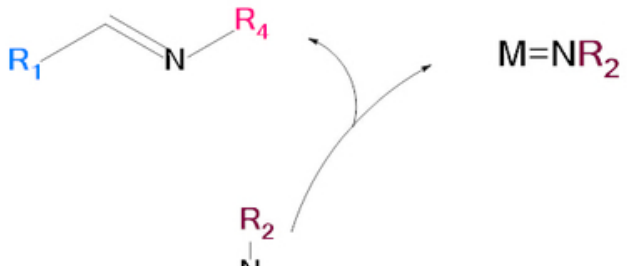

$$
\text { N }
$$
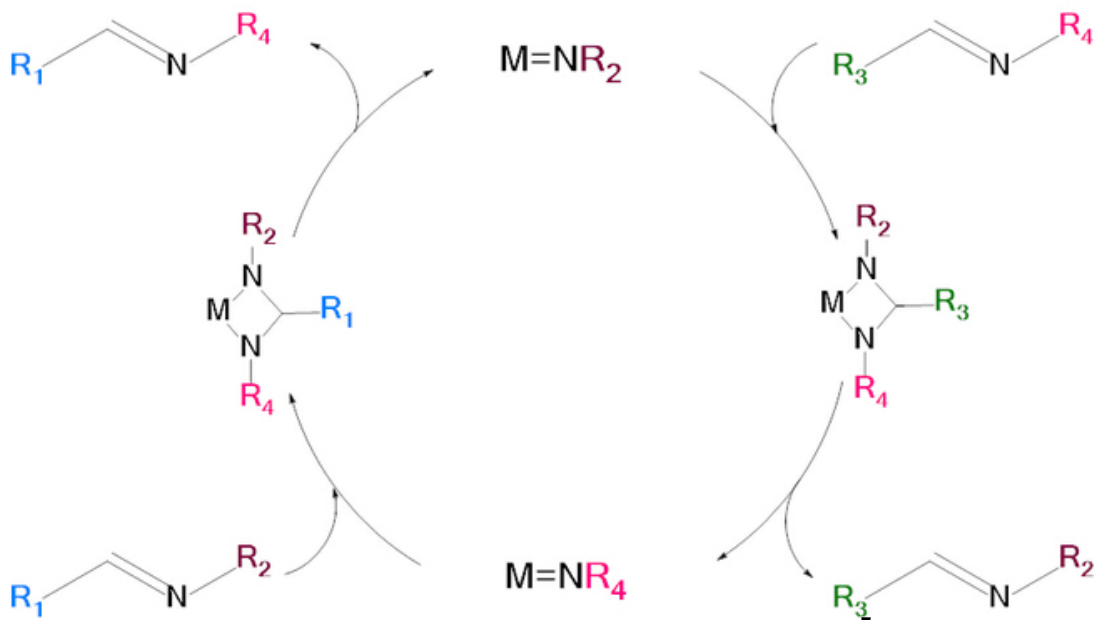

Figure 2: General reaction scheme of the imine metathesis mechanism ${ }^{7}$. Please click here to view a larger version of this figure.

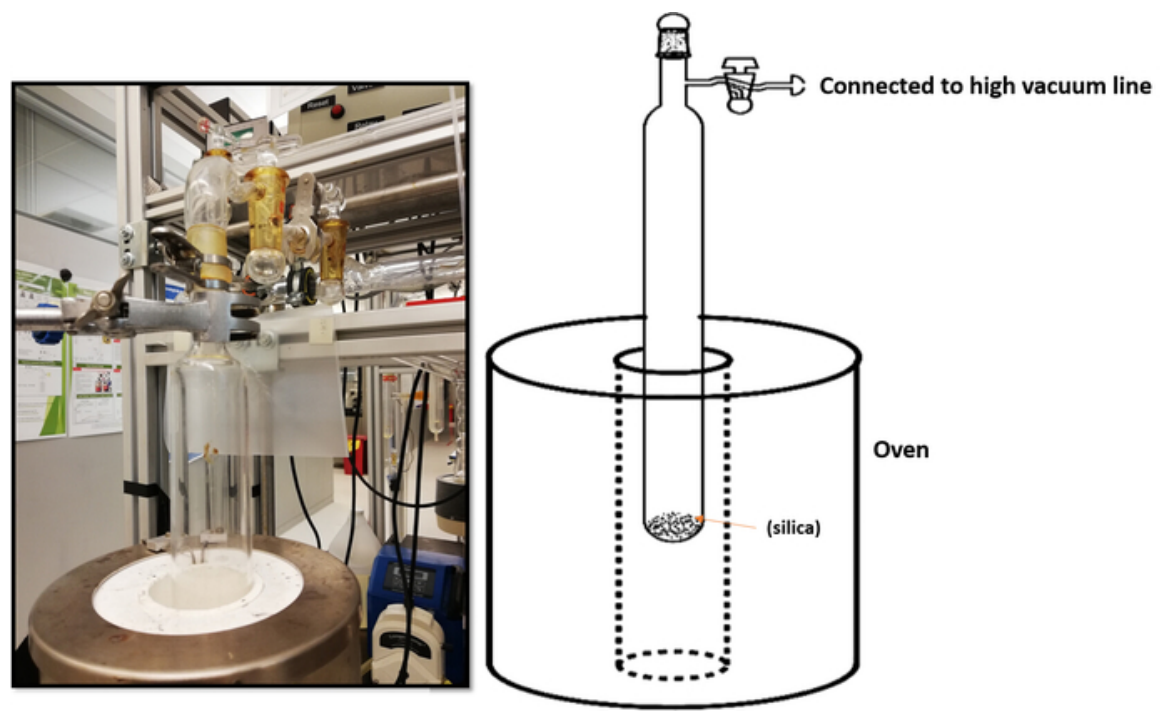

Figure 3: Dehydroxylation of silica. The reactor was inserted into the oven and connected to a high vacuum line (HVL). The photograph shows the actual setup. Please click here to view a larger version of this figure.
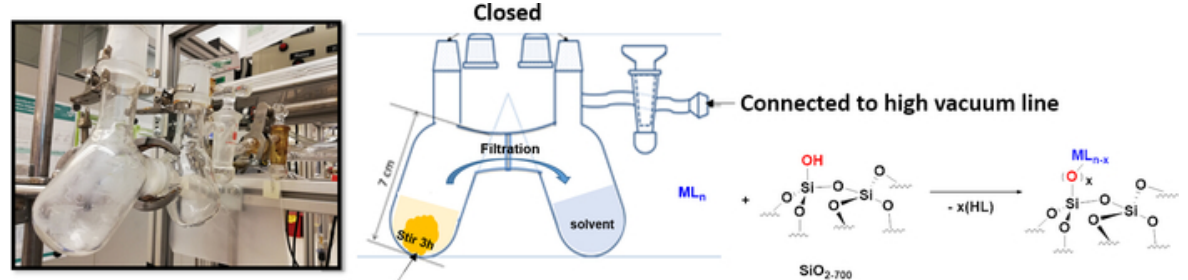

$\left(\mathrm{SiO}_{2-700}+\right.$ Complex)

in Pentane as solvent

Figure 4: Grafting in a double Schlenk. Schematic representation of the grafting process. The photograph shows the actual setup. Please click here to view a larger version of this figure. 
Solvent Transfer by Distillation

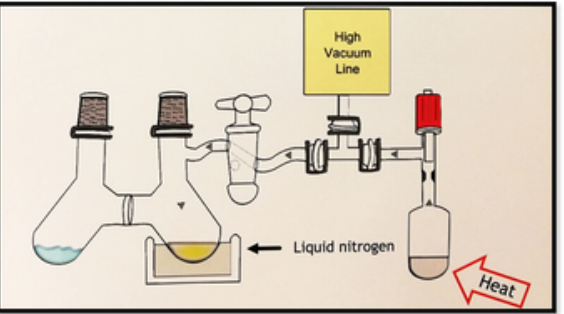

Solvent Removal Using Interceptor

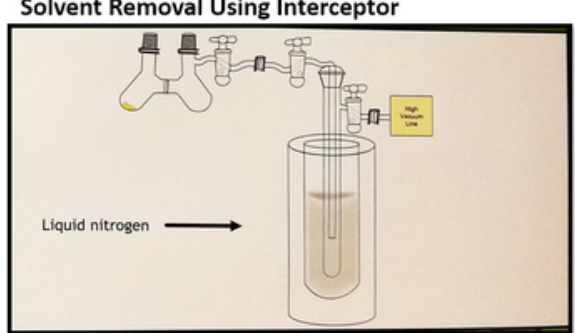

Washing Cycle

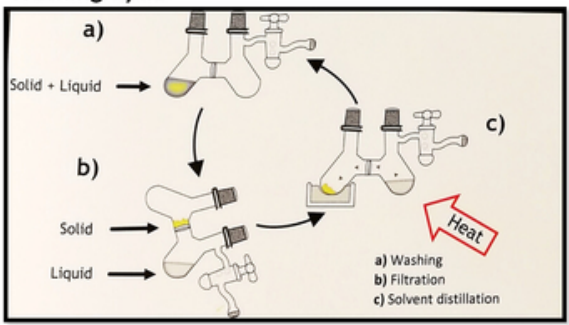

Final Drying

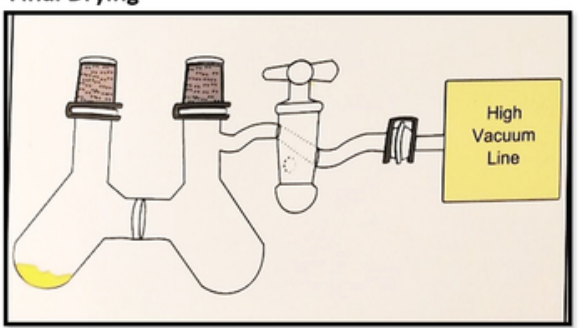

Figure 5: Handling grafting in a double Schlenk with a high vacuum line (HVL). First, the solvent transfer procedure was performed, followed by the washing procedure, the solvent removal, and drying the material. Please click here to view a larger version of this figure.

A

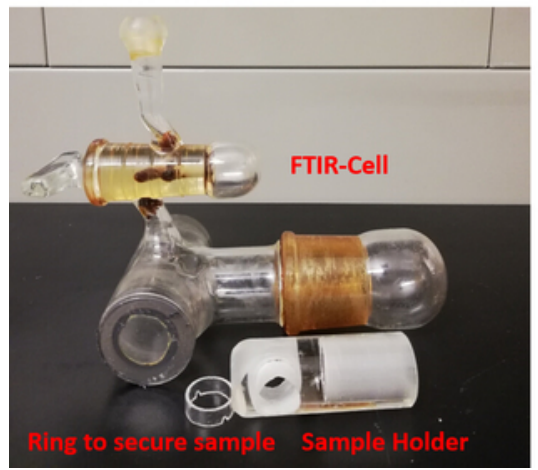

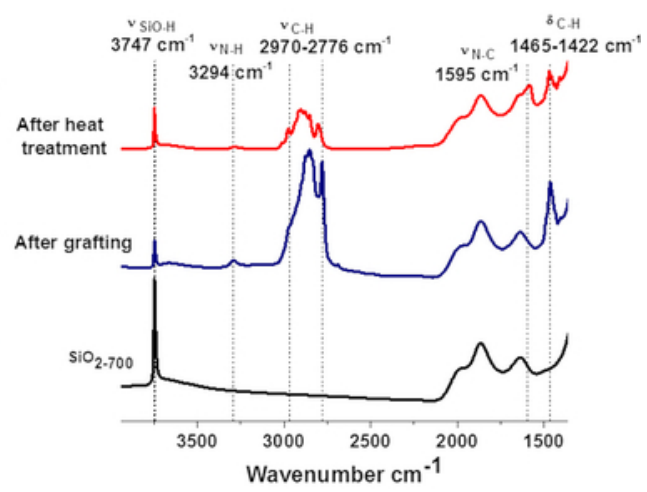

Figure 6: Fourier-transform infrared spectroscopy (FTIR) measurement. (A) FTIR disc pellet preparation. (B) FTIR spectra of the support, $\mathrm{SiO}_{2-700}$ have a characteristic signal for isolated silanols observed at $3,747 \mathrm{~cm}^{-1}$ and silica undertone between $1,400-2000 \mathrm{~cm}^{-1}$. For the grafted complex, strong new signals appeared in the regions of $2,800-3,000 \mathrm{~cm}^{-1}$ and $1,400-1,500 \mathrm{~cm}^{-1}$. They represent the alkyl groups. After heat treatment, spectrum shows a new signal appeared at $1,595 \mathrm{~cm}^{-1}$ for the imido group ${ }^{7}$. Please click here to view a larger version of this figure. 
A)

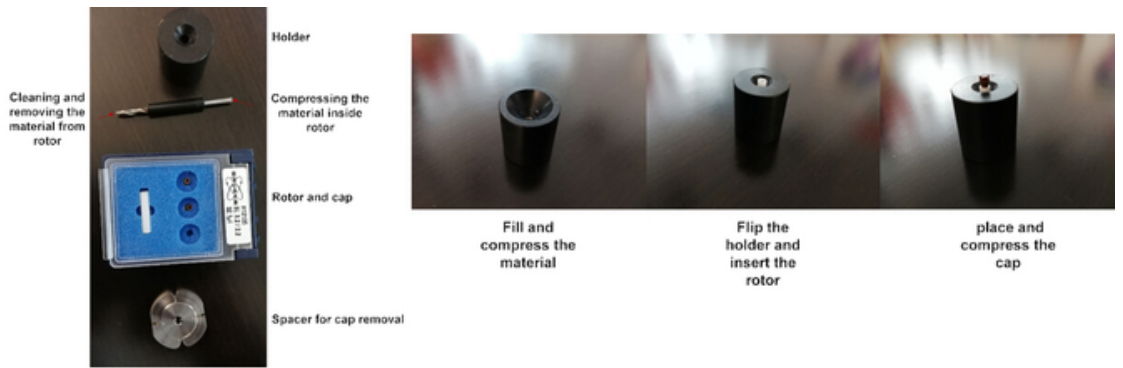

B)

C)

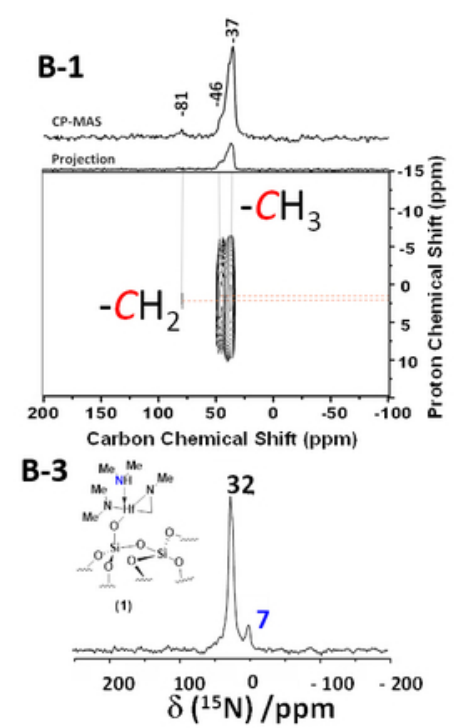

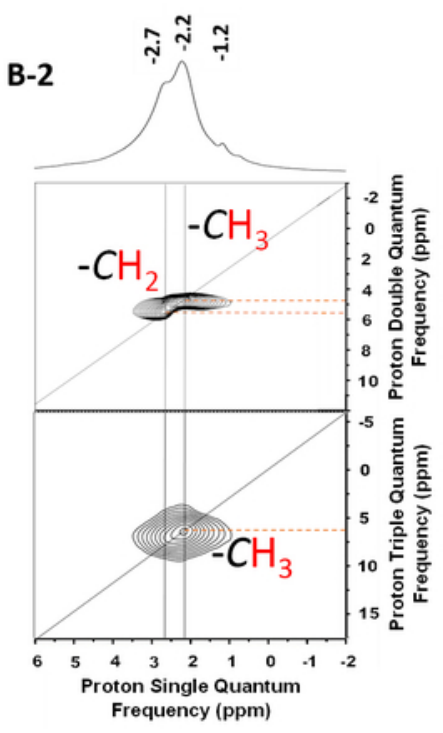

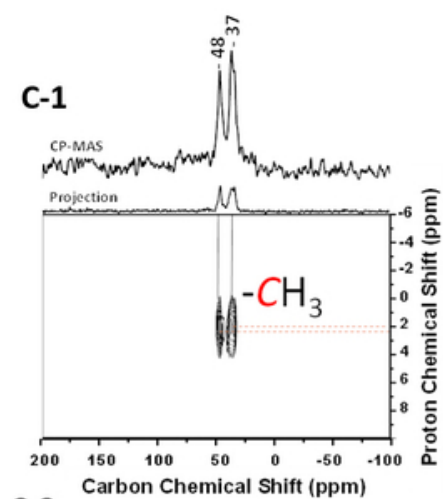

C-3 me
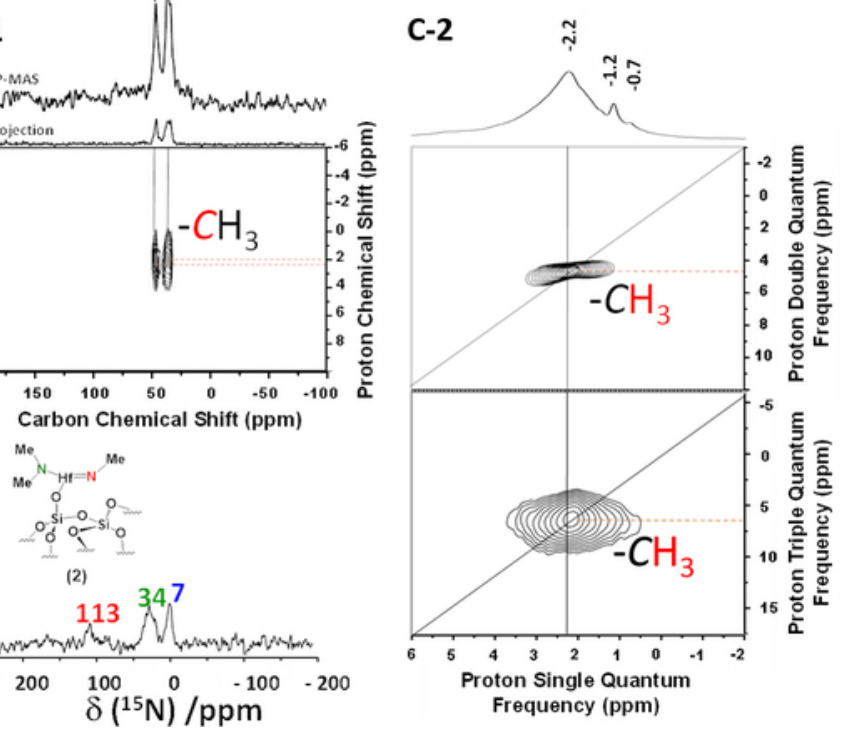

Figure 7: NMR data from the grafted material and the catalyst. (A) Preparing a solid-state NMR sample. (B) (B-1) $1 \mathrm{D}^{13} \mathrm{C}$ cross-polarized magic angle spinning (CP-MAS) with $2 \mathrm{D}^{1} \mathrm{H}-{ }^{13} \mathrm{C}$ heteronuclear correlation (HETCOR( NMR spectra of the grafted complex before treatment. (B-2) $1 D^{1} \mathrm{H}$ NMR spectrum with ${ }^{1} \mathrm{H}-{ }^{1} \mathrm{H}$ double-quantum (DQ) and ${ }^{1} \mathrm{H}-{ }^{1} \mathrm{H}$ triple quantum (TQ) spectra of the grafted complex. (B-3) ${ }^{15} \mathrm{~N}$ dynamic nuclear polarization surface enhanced the NMR spectroscopy (DNP-SENS) spectrum and (inset 1) the proposed structure of the grafted surface hafnium complex $\left[(\equiv \mathrm{Si}-\mathrm{O}-) \mathrm{Hf}\left(\eta^{2}-\mathrm{MeNCH}_{2}\right)\left(\eta^{1}-\mathrm{NMe}_{2}\right)\left(\eta^{1}-\mathrm{HNMe}_{2}\right)\right]$. (C) (C-1) $1 \mathrm{D}^{13} \mathrm{C}$ CP-MAS with $2 \mathrm{D}{ }^{1} \mathrm{H}-{ }^{13} \mathrm{C}$ HETCOR NMR spectra of the grafted complex after heat treatment to generate an imido fragment. $(\mathbf{C}-2) 1 \mathrm{D}^{1} \mathrm{H}$ NMR spectrum with ${ }^{1} \mathrm{H}-{ }^{-1} \mathrm{H}$ DQ and ${ }^{1} \mathrm{H}-{ }^{1} \mathrm{H}$ TQ spectra of the grafted complex after heat treatment which is the catalyst 2. (C-3) ${ }^{15} \mathrm{~N}$ DNP-SENS spectrum, and (inset 2) proposed a structure of the surface hafnium complex catalyst $\left[(\equiv \mathrm{Si}-\mathrm{O}-) \mathrm{Hf}(=\mathrm{NMe})\left(\eta^{1}-\mathrm{NMe}_{2}\right)\right]^{3}$. Please click here to view a larger version of this figure. 

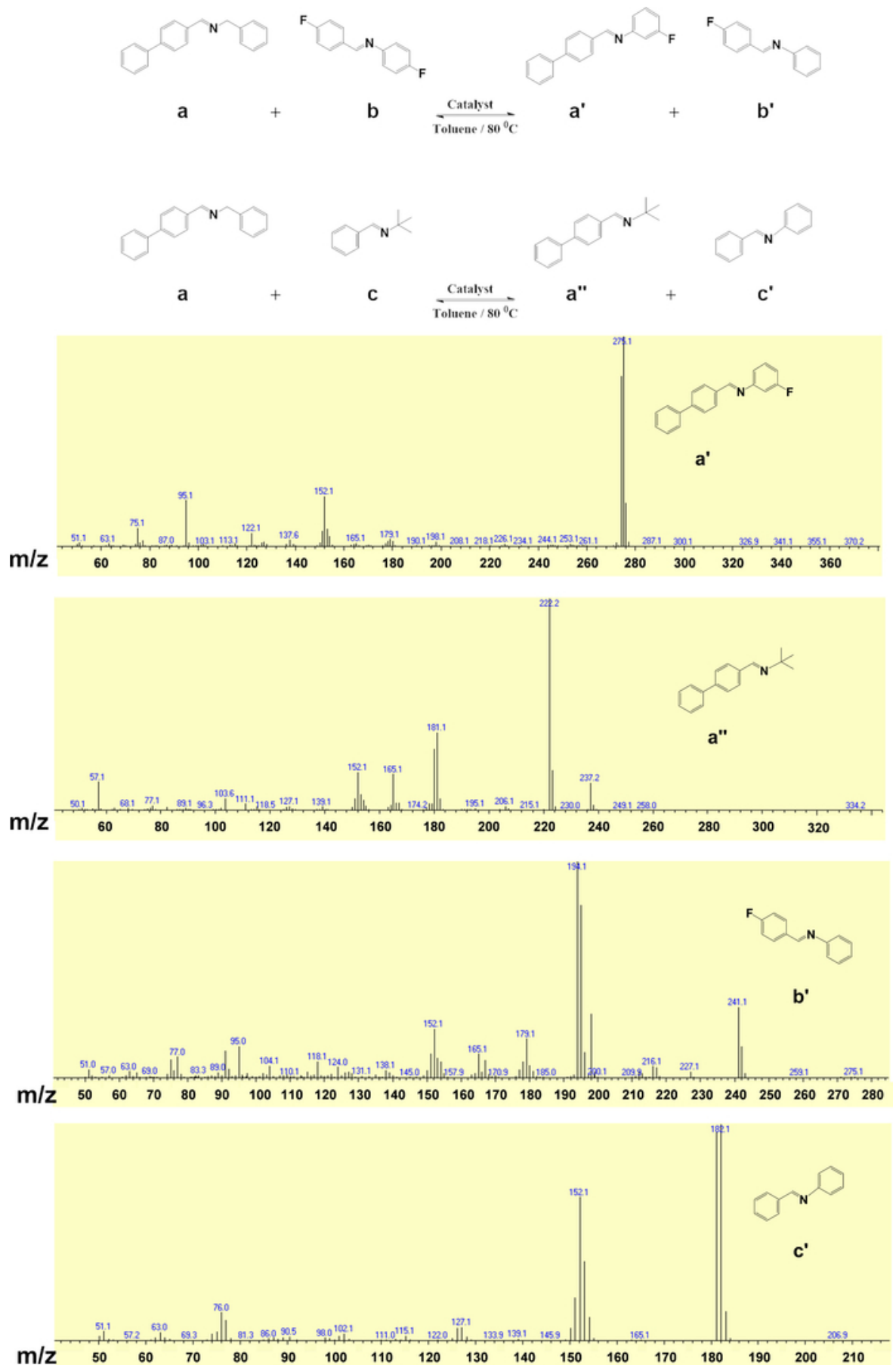

Figure 8: Imine metathesis catalysis and mass spectral data of products. Three imine compounds were tested: $\mathrm{N}$-(4-

phenylbenzylidene)benzylamine (a), N-(4-fluorobenzylidene)-4-fluoroaniline (b), and N-benzylidene-tert-butylamine (c). Gas chromatographymass spectrometry (GC-MS) was used to analyze the products obtained, namely 1-([1,1'-biphenyl]-4-yl)-N-(tert-butyl)methanimine (a"), $\mathrm{N}, 1$ -

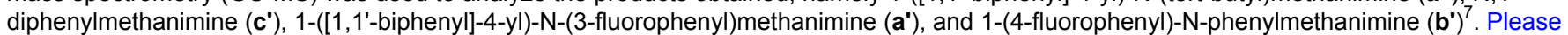
click here to view a larger version of this figure.

\begin{tabular}{|l|l|l|l|l|l|l|l|}
\hline & Metal & \%Metal & M/Silanol & \% & \%N & C/N & N/M \\
\hline $\mathbf{1}$ & Hf & 4.94 & 0.92 & 2.49 & 1.5 & 2 & 3.9 \\
\hline $\mathbf{2}$ & Hf & 4.48 & 0.81 & 1.4 & 0.8 & 1.8 & 7.1 \\
\hline
\end{tabular}

Table 1: Elemental analysis ${ }^{7}$. 


\begin{tabular}{|l|l|l|l|}
\hline Catalyst & Time & substrate conversion \% & Substrate conversion \% \\
\hline $\mathbf{2}$ & 1 & $\mathrm{a}(54)$ & $\mathrm{c}(54)$ \\
\hline no catalyst & 1 & $\mathrm{a}(11)$ & $\mathrm{c}(11)$ \\
\hline $\mathbf{2}$ & 6 & $\mathrm{a}(50)$ & $\mathrm{b}(55)$ \\
\hline no catalyst & 6 & $\mathrm{a}(25)$ & $\mathrm{b}(20)$ \\
\hline $\mathbf{2}$ & 4 & $\mathrm{a}(36)$ & $\mathrm{b}(30)$ \\
\hline
\end{tabular}

Table 2: Catalysis Conversion ${ }^{7}$.

\section{Discussion}

The methodology employed in SOMC was developed to handle sensitive materials (such as highly dehydroxylated silica and sensitive complexes precursors, etc.) in the cleanest way possible. This is necessary to prepare and characterize single-site well-defined surface complexes. Furthermore, these complexes can be isolated and serve as intermediates to various catalytic transformations of interest (e.g., alkane metathesis ${ }^{4,5}$, imine metathesis ${ }^{7,8,32}$, and hydroaminoalkylation ${ }^{26}$ ).

HVLs (producing a vacuum as high as $10^{-5} \mathrm{mbar}$ ) are necessary to avoid any contamination from inert gases during the grafting reactions. High vacuum techniques are very different from the positive pressure techniques employed in regular Schlenk lines or even gloveboxes. Due to its high surface area ( $200 \mathrm{~m}^{2}$ per gram), silica tends to adsorb contaminants (water, etc.) that can compromise subsequent reactions.

The glovebox is used mostly to load/unload reactants prior to the reactions and to conduct some reactions. All gloveboxes in this method operate under argon as some catalysts can react with nitrogen. The method requires special attention dedicated to transferring sensitive materials from gloveboxes to HVLs and back. Some steps (i.e., liquid addition and solid washing) require the user to assemble several pieces of glassware on the HVL. Each connection must remain leak-free for the time of the operation (i.e., during the solvent transfer) to protect the chemicals contained.

This technique is rather time-consuming and challenging to upscale but remains unparalleled in producing a significant amount (1-3 g) of clean and well-characterized surface complexes that can be used as catalysts. Future development foreseen is the use of this method to isolate more new catalytic species, such as those containing SOMFs (i.e., metal-nitrido fragment $M \equiv N$ ).

In this study, a SOMF metal amide fragment was isolated. Highly dehydroxylated silica $\mathrm{SiO}_{2-700}$ was treated with about one equivalent of metal precursor $\left(\mathrm{Hf}\left(\mathrm{NMe}_{2}\right)_{4}[0.089 \mathrm{~mL}]\right)$ to the amount of silanol $\left(0.3 \mathrm{mmol} \cdot \mathrm{g}^{-1}\right)$ in pentane at room temperature to afford catalysts $\mathrm{s}^{7,8,32}$. The volatiles were removed, and the powder samples were dried under a dynamic vacuum to afford the desired materials, which were heated to $200{ }^{\circ} \mathrm{C}$ to generate imido. All materials should be characterized by FTIR, elemental microanalysis, and SSNMR and DNP-SENS.

The surface silanols were almost completely consumed as evidenced by the disappearing of the signal at $3,747 \mathrm{~cm}^{-1}$ in the FTIR spectra of the grafted complexes. New signals observed around $2,800-3,000 \mathrm{~cm}^{-1}$ and $1,400-1,500 \mathrm{~cm}^{-1}$ correspond to alkyl groups. Further studies by CHNS and ICP analysis provide information about the metal loading for complex $1(4.49 \%$ of $\mathrm{Hf}$ in weight per gram of silica $(0.91 \mathrm{mmol}$ of $\mathrm{Hf}$ per silanol)) that is consistent with a monopodial species. The expected and found $\mathrm{M} / \mathrm{C}, \mathrm{M} / \mathrm{H}$, and $\mathrm{M} / \mathrm{N}$ ratios are $\mathrm{N} / \mathrm{M}=3.9($ theory $=3)$ and $\mathrm{C} / \mathrm{M}=$ 7.1 (theory = 6) for the grafted material 1 (see Table 1); for 2 , the N/M and C/M ratios are 2.5 (theory $=2$ ) and 4.6 (theory $=4$ ), respectively.

To get an insight into the coordination sphere of the surface grafted metal complexes, the materials were examined by advanced SSNMR spectroscopy experiments and DNP-SENS studies. SSNMR results are generally more difficult to interpret compared to liquid NMR spectroscopy; SSNMR is for insoluble macromolecules. Solid samples have less isotropic molecular tumbling compared to liquid samples; the molecules can tumble in all directions as they are homogeneous and diamagnetic, which will result in much broader signals in SSNMR for solid samples ${ }^{40}$.

From DQ SSNMR, we could observe $-\mathrm{CH}_{2}$ and $-\mathrm{CH}_{3}$, but from TQ SSNMR, only $-\mathrm{CH}_{3}$ was presented, and from HETCOR correlation, we could elucidate the structure. We focused on the metal-nitrogen fragment which was delivered by the use of ${ }^{15} \mathrm{~N}$ DNP-SENS, since we were working on amine complexes.

To conclude, the preparation of new silica-supported hafnium complexes [( $\left.=\mathrm{Si}-\mathrm{O}-) \mathrm{Hf}\left(\eta^{2}-\mathrm{MeNCH}_{2}\right)\left(\eta^{1}-\mathrm{NMe}_{2}\right)\left(\eta^{1}-\mathrm{HNMe} \mathrm{H}_{2}\right)\right]$ 1, and $[(\equiv \mathrm{Si}-$ $\left.\mathrm{O}-) \mathrm{Hf}(=\mathrm{NMe})\left(\eta^{1}-\mathrm{NMe}_{2}\right)\right] 2$ fully characterized by SS NMR and FTIR spectroscopies and elemental analysis. Further characterizations were carried giving structural information about nitrogen atom by ${ }^{15} \mathrm{~N}$ MAS DNP SENS data. The data show the presence of surface fragments (MNC) in 1 , and $([M]=N)$ fragment in 2 . The essential role of $([M]=N R)$ fragment in imine metathesis was demonstrated in catalyst testing with two pairs of imine substrates.

\section{Disclosures}

The authors have nothing to disclose.

\section{Acknowledgments}

The authors would like to express their thanks to the King Abdullah University of Science and Technology (KAUST) for financial and human support. 


\section{References}

1. Woo, L. K. Intermetal oxygen, sulfur, selenium, and nitrogen atom transfer reactions. Chemical Reviews. 93 (3), $1125-1136$ (1993).

2. Bruno, J. W., Li, X. J. Use of Niobium (III) and Niobium (V) Compounds in Catalytic Imine Metathesis under Mild Conditions. Organometallics. 19 (23), 4672-4674 (2000).

3. Sun, M. et al. Catalytic oxidation of light alkanes (C1-C4) by heteropoly compounds. Chemical Reviews. 114 (2), $981-1019$ (2013).

4. Basset, J.-M., Coperet, C., Soulivong, D., Taoufik, M., Cazat, J. T. Metathesis of alkanes and related reactions. Accounts of Chemical Research. 43 (2), 323-334 (2009).

5. Blanc, F., Copéret, C., Thivolle-Cazat, J., Basset, J.-M. Alkane Metathesis Catalyzed by a Well-Defined Silica-Supported Mo Imido Alkylidene Complex: [(=SiO)Mo(=NAr) $(=\mathrm{CHtBu})(\mathrm{CH} 2 \mathrm{tBu})]$. Angewandte Chemie International Edition. 45 (37), 6201-6203 (2006).

6. Riache, N. et al. Striking difference between alkane and olefin metathesis using the well-defined precursor [[triple bond, length as $\mathrm{m}$-dash] $\mathrm{Si}-\mathrm{O}-\mathrm{WMe}$ 5]: indirect evidence in favour of a bifunctional catalyst W alkylidene-hydride. Catalysis Science \& Technology. 5 (1), 280-285 (2015).

7. Aljuhani, M. Imine Metathesis Catalyzed by a Silica-Supported Hafnium Imido Complex. ACS Catalysis. 8 (10), $9440-9446$ (2018).

8. Barman, S. et al. Well-Defined Silica Grafted Molybdenum Bis(imido) Catalysts for Imine Metathesis Reactions. Organometallics. 36 (8), 1550-1556 (2017).

9. Hamzaoui, B. Well-defined silica-supported zirconium-imido complexes mediated heterogeneous imine metathesis. Chemical Communications. 52 (25), 4617-4620 (2016).

10. Mclnnes, J. M. Transition metal imide/organic imine metathesis reactions: unexpected observations. Chemical Communications. 0 (16), 1669-1670 (1998).

11. Gibson, V. C. Metathesis Polymerization: ROMPing towards new materials. Advanced Materials. 6 (1), 37-42 (1994).

12. Nugent, W. A., Haymore, B. L. Transition metal complexes containing organoimido (NR) and related ligands. Coordination Chemistry Reviews. 31 (2), 123-175 (1980).

13. Duncan, A. P., Bergman, R. G. Selective transformations of organic compounds by imidozirconocene complexes. The Chemical Record. 2 (6), 431-445 (2002).

14. Schrock, R. R., Hoveyda, A. H. Molybdenum and tungsten imido alkylidene complexes as efficient olefin-metathesis catalysts. Angewandte Chemie International Edition. 42 (38), 4592-4633 (2003).

15. Bolton, P. D., Mountford, P. Transition metal imido compounds as Ziegler-Natta olefin polymerisation catalysts. Advanced Synthesis \& Catalysis. 347 (2-3), 355-366 (2005).

16. Pelletier, J. D., Basset, J.-M. Catalysis by Design: Well-Defined Single-Site Heterogeneous Catalysts. Accounts of Chemical Research. 49 (4), 664-677 (2016).

17. Banks, R. L., Bailey, G. C. Olefin Disproportionation. A New Catalytic Process. Industrial \& Engineering Chemistry Product Research and Development. 3 (3), 170-173 (1964).

18. Balcar, H., Čejka, J. Mesoporous molecular sieves as advanced supports for olefin metathesis catalysts. Coordination Chemistry Reviews. 257 (21-22), 3107-3124 (2013).

19. Mol, J. C. Olefin metathesis over supported rhenium oxide catalysts. Catalysis Today. 51 (2), 289-299 (1999).

20. Buchowicz, W., Ingold, F., Mol, J. C., Lutz, M., Spek, A. L. Novel Ruthenium (II) 2 Carboxylates as Catalysts for Alkene Metathesis. Chemistry-A European Journal. 7 (13), 2842-2847 (2001).

21. Casey, C. P. 2005 Nobel Prize in Chemistry. Development of the Olefin Metathesis Method in Organic Synthesis. Journal of Chemical Education. 83 (2), 192 (2006).

22. Poater, A., Solans-Monfort, X., Clot, E., Copéret, C., Eisenstein, O. Understanding d0-Olefin Metathesis Catalysts: Which Metal, Which Ligands? Journal of the American Chemical Society. 129 (26), 8207-8216 (2007).

23. Vidal, V., Théolier, A., Thivolle-Cazat, J., Basset, J.-M. Metathesis of Alkanes Catalyzed by Silica-Supported Transition Metal Hydrides. Science. 276 (5309), 99-102 (1997).

24. Le Roux, E. et al. Development of Tungsten-Based Heterogeneous Alkane Metathesis Catalysts Through a Structure-Activity Relationship. Angewandte Chemie. 117 (41), 6913-6916 (2005).

25. Goldman, A. S. et al. Catalytic alkane metathesis by tandem alkane dehydrogenation-olefin metathesis. Science. 312 (5771), 257-261 (2006).

26. Hamzaoui, B. et al. Isolation and Characterization of Well-Defined Silica-Supported Azametallacyclopentane: A Key Intermediate in Catalytic Hydroaminoalkylation Reactions. Advanced Synthesis \& Catalysis. 357 (14-15), 3148-3154 (2015).

27. Schafer, L., Chong, E., Garcia, P. Hydroaminoalkylation: Early-Transition-Metal-Catalyzed a-Alkylation of Amines. Synthesis. 46 (21), 2884-2896 (2014).

28. Müller, T. E., Hultzsch, K. C., Yus, M., Foubelo, F., Tada, M. Hydroamination: Direct Addition of Amines to Alkenes and Alkynes. Chemical Reviews. 108 (9), 3795-3892 (2008).

29. Ciaccia, M., Di Stefano, S. Mechanisms of imine exchange reactions in organic solvents. Organic \& Biomolecular Chemistry. 13 (3), $646-654$ (2015).

30. Ingold, C. K., Piggott, H. A. CCLXXXVII.-The mobility of symmetrical triad systems. Part I. The conditions relating to systems terminated by phenyl groups. Journal of the Chemical Society: Transactions. 121 (0), 2381-2389 (1922).

31. Tóth, G., Pintér, I., Messmer, A. Mechanism of the exchmge reaction of aromatic scriff bases. Tetrahedron Letters. 15 (9), $735-738$ (1974).

32. Hamzaoui, B., Pelletier, J. D. A., Abou-Hamad, E., Basset, J.-M. Well-defined silica-supported zirconium-imido complexes mediated heterogeneous imine metathesis. Chemical Communications. 52 (25), 4617-4620 (2016).

33. Hamzaoui, B., Pelletier, J. D., Abou-Hamad, E., Basset, J.-M. Well-defined silica-supported zirconium-imido complexes mediated heterogeneous imine metathesis. Chemical Communications. 52 (25), 4617-4620 (2016).

34. Müller, C., Saak, W., Doye, S. Neutral Group-IV Metal Catalysts for the Intramolecular Hydroamination of Alkenes. European Journal of Organic Chemistry. 2008 (16), 2731-2739 (2008).

35. Pohlki, F., Doye, S. The catalytic hydroamination of alkynes. Chemical Society Reviews. 32 (2), 104-114 (2003).

36. Cook, A. K., Copéret, C. Alkyne Hydroamination Catalyzed by Silica-Supported Isolated Zn(II) Sites. Organometallics. 37 (9), $1342-1345$ (2018). 
37. Prochnow, I., Zark, P., Müller, T., Doye, S. The Mechanism of the Titanium-Catalyzed Hydroaminoalkylation of Alkenes. Angewandte Chemie International Edition. 50 (28), 6401-6405 (2011).

38. Brunet, J. J., Neibecker, D. Catalytic Hydroamination of Unsaturated Carbon-Carbon Bonds. Catalytic Heterofunctionalization. 91-141 (2001).

39. El Eter, M., Hamzaoui, B., Abou-Hamad, E., Pelletier, J. D., Basset, J.-M. Well-defined azazirconacyclopropane complexes supported on silica structurally determined by 2D NMR comparative elucidation. Chemical Communications. 49 (41), 4616-4618 (2013).

40. Duer, M. J. Solid state NMR spectroscopy: principles and applications. John Wiley \& Sons (2008). 